QUIPURAMAYOC $\mid$ Revista de la Facultad de Ciencias Contables

Vol. 20 N. ${ }^{\circ} 38$ pp. 102-108 (2012) UNMSM, Lima, Perú

ISSN: 1560-9103 (versión impresa) / ISSN: 1609-8196 (versión electrónica)

\title{
LA CONTABILIDAD CREATIVA Y SU IMPACTO EN LA INFORMACIÓN FINANCIERA
}

CREATIVE ACCOUNTING AND ITS IMPACT ON THE FINANCIAL INFORMATION

\author{
Dorie Cruz Ramírez* \\ Suly Sendy Pérez Castañeda* \\ Victor Manuel Piedra Mayorga*** \\ Docente Investigador de la Escuela Superior de Cd. Sahagún \\ Universidad Autónoma del Estado de Hidalgo-México \\ [Recepción: Setiembre de 2012/ Conformidad: Octubre de 2012]
}

\section{RESUMEN}

La manipulación contable o contabilidad creativa es un tema de actualidad, es una práctica muy extendida pero poco combatida: la alteración de beneficios. Actividad que se ha realizando durante años por diversos países, como si de un juego se hablase, lo cierto es que su naturaleza y consecuencias es un tema máximo interés, puesto que una elección contable oportunista por parte de la gerencia de una empresa puede acarrear que determinados individuos sean beneficiados en detrimento de otros. La forma en que se esconde la deuda fuera de un balance, cómo alterar las partidas de ingresos y costos. Según una investigación de la Securities and Exchange Commission de los EEUU, más de la mitad de los abusos contables de los 90's se debieron a políticas incorrectas de reconocimiento de ingresos. La influencia de la manipulación contable sobre la estructura financiera y patrimonial repercute sobre la veracidad de la información financiera contable y por tanto sobre la valoración de la unidad económica. La subjetividad de los diferentes tratamientos contables a la hora de determinar la información financiera es crucial para dar pie a abusos y cómo las diferentes políticas de consolidación han sido usadas y abusadas hasta generar importantes escándalos contables.

Palabras claves: Contabilidad creativa, Información financiera, Control interno y Gobierno corporativo.

\begin{abstract}
The accounting manipulation or creative accounting is a current topic, it is widely practiced but little combated: altering benefits. Activity has been done for years by various countries, as if it were a game, the truth is that its nature and consequences is a topic of great interest, since an opportunistic accounting choice taken by the management of a company can lead to certain individuals are privileged at the expense of others. How to hide debt off balance, how to alter items of income and expenses. According to an investigation by the Securities and Exchange Commission in the U.S., more than half of the financial abuse of the 90's were due to incorrect policies for revenue recognition. The influence of accounting manipulation on financial and equity structure has an impact on the truth of financial reporting and thus accounting for the valuation of the economic unit. The subjectivity of different accounting treatments for determining the financial information is crucial to be open to abuse and how different consolidation policies have been used and abused until to generate significant accounting scandals.
\end{abstract}

Keywords: Creative accounting, Financial information, Internal control, Corporate governance.

* Licenciada en Contaduría. E-mail. doriec@uaeh.edu.mx

** Licenciada en Contaduría y Finanzas, así como Licenciada en Ciencias de la Comunicación, México. E-mail.sulysp@uaeh.edu.mx.

*** Licenciado en Administración de Empresas. E-mail piedrinix@uaeh.edu.mx 


\section{METODOLOGÍA}

Investigación exploratoria, debido a que su objetivo radica en la obtención de un conocimiento y la realización de un breve análisis sobre conceptos contables como son información financiera, ética profesional, gobierno corporativo, contabilidad creativa, bajo la perspectiva de una investigación documental de artículos realizados por expertos en la materia, dentro del proyecto de investigación denominado Tendencias Contables en México.

\section{INTRODUCCIÓN}

La contabilidad creativa consiste fundamentalmente en aprovechar los vacíos de la normativa legal en cuanto a las diferentes posibilidades de elección con el propósito de transformar la información contable que se presentan en los estados financieros de las empresas, aún cuando la contabilidad, desde el punto normativo como lo establece el IMCP y para lo cual se creó, tiene como objetivo proporcionar información financiera a los diversos interesados en términos relevantes, que permitan y favorezcan la toma de decisiones.

Pero en algunos casos es común la afirmación de que "La cuenta de resultados de una empresa comienza desde abajo". Hace referencia a la práctica de establecer el beneficio que espera el mercado, de una empresa, y a partir de ahí "maquillar" los costos y las ventas hasta hacer que el neto coincida con la cifra de beneficios deseada.

Los diferentes mecanismos para reducir ficticiamente el nivel de deuda de un balance, con el objetivo de contentar a auditores, a mercados de deuda, y a los de fondos propios de la empresa.

Con lo anterior expuesto nos preguntamos:
¿Qué falla en los mecanismos de control de una empresa para que se generen los abusos contables? ¿Qué medidas corresponden aplicar en una organización para prevenir un abuso contable o delito fiscal?

Será acaso: ¿Falta de responsabilidad social corporativa? ¿Un adecuado estudio de técnicas de buen gobierno corporativo que no han prevenido la formación de escándalos contables? ¿Falta de ética profesional $i \underset{ }{i} \mathrm{O}$ una combinación de todas y cada una de las anteriores?

\section{ALGUNOS ASPECTOS HISTÓRICOS}

La contabilidad es considerada una de las áreas del conocimiento de mayor antigüedad a nivel mundial, como se puede observar en lugares como son Mesopotamia, Egipto, Grecia, Roma, entre otros, donde se tienen indicios de esta área debido al nivel de actividades comerciales que realizaban.

El primer autor del que se tiene conocimiento que estableció claramente el uso del método de la partida doble Benedetto Contrugli. El libro de Contrugli tardó casi 115 años en ser llevado a la imprenta, lo que, unido al carácter incompleto de su exposición impide que se pueda adjudicar a su autor en la historia de la contabilidad, un papel comparable al del Monje Franciscano Fray Luca Pacioli, su obra de tuvo gran éxito, particularmente la parte dedicada a la práctica comercial y contable, que fue reimpresa por separado algunos años mas tarde.

Derivado de esta área del conocimiento surge la contaduría pública, que es considerada una profesión multidisciplinaria, que goza de ser complementada por otras áreas del conocimiento de vital importancia como son: el derecho, la economía, la administración, las matemáticas, actualmente la informática que ha dado mucho realce y actuali- 
dad al registro contable, dándole al contador público una formación integral que le permita desempeñarse, en distintos sectores económicos, ocupando diversidad de puestos y cargos dentro de las entidades económicas, donde tiene que demostrar sus habilidades, actitudes y aptitudes de trabajo.

En la actualidad, la contabilidad ha tomado una nueva faceta, la tarea informativa ya no es el único objetivo que la enmarca, la nueva realidad económica ha hecho que la profesión contable tome una gran importancia dentro del marco empresarial actual.

En el estudio de la contabilidad han surgido nuevas expectativas y nuevos cuestionamientos sobre la orientación que se le pueda estar dando a esta profesión a causa de los rápidos cambios económicos, tecnológicos y sociales de la actualidad.

Estas modificaciones han dado paso a nuevas formas de ver y analizar el papel de los contadores dentro de las empresas; ya no solo como el técnico de un proceso de recolección y generador de información, sino como un pensador que aporta con investigación y conocimiento, ideas que ayudarán a mejorar el funcionamiento de las organizaciones y por ende el fundamentar una nueva técnica contable que se adapte al nuevo orden mundial.

Por todo lo anterior expuesto y haciendo referencia que en la actualidad se está perdiendo el fin para lo cual se creó la contabilidad, surge este tema de investigación en relación a la contabilidad creativa y su relación con la información financiera.

\section{CONCEPTUALIZACIONES}

El Consejo Mexicano para a investigación y Desarrollo de las Normas de Información Financiera (CINIF), emite las Normas de Información Financiera (NIF), donde se conceptualiza que es la Contabilidad en las NIF A-1 (2008) párrafo 3 y la conceptualiza como "Una técnica que se utiliza para el registro de las operaciones que afectan económicamente a una entidad y que produce sistemática y estructuradamente información financiera. Las operaciones que afectan a una entidad incluyen las transacciones, transformaciones internas y otros eventos."

Así mismo el concepto de Información Financiera que también se encuentra fundamentado en las NIF A-1 (2008) párrafo 4 establece que:

"La información financiera que emana de la contabilidad, es información cuantitativa, expresada en unidades monetarias y descriptiva, que muestra la posición y desempeño financiero de una entidad, y cuyo objetivo esencial es el de ser útil al usuario general en la toma de sus decisiones económicas. Su manifestación fundamental son los estados financieros. Se enfoca esencialmente a proveer información que permita evaluar el desenvolvimiento de la entidad, así como proporcionar elementos de juicio para estimar el comportamiento futuro de los flujos de efectivo, entre otros aspectos."

Por lo tanto existen múltiples participantes en la actividad económica y sus intereses son muy diversos y complejos, lo que con lleva que la información financiera sea de uso y aplicación general, que satisfaga los requerimientos de información a todos y cada uno de los diversos interesados.

Por otro lado tenemos a la Ética profesional que de acuerdo al Instituto Mexicano de Contadores Públicos (IMCP), y con el objeto de ofrecer mayores garantías de solvencia moral y establecer normas de actuación profesional, dicho instituto emitió un Código de Ética Profesional.

El Código de Ética profesional (2009), establece que "La necesidad de tener un 
código, nace de la aplicación de las normas generales de conducta en la práctica diaria. Puesto que todos los actos humanos son regidos por la ética, también las reglas escritas deben ser de aplicación general."

Un Código de Ética Profesional, no sólo sirve de guía a la acción moral; también, mediante él, la profesión declara su intención de cumplir con la sociedad; con quien ampara las relaciones de trabajo o dicho de otra forma quien patrocina de sus servicios; y hacia la misma profesión contable con el fin de lograr en su conjunto un trabajo ético y de calidad.

Pasamos a la conceptualización de Gobierno Corporativo y para tal situación el autor López, V. (2009) "El gobierno corporativo es el conjunto esencial de acciones directivas seguidas por la organización, a fin de lograr razonabilidad, rendición de cuentas, transparencia y responsabilidad".

El gobierno corporativo juega un papel importante y fundamental al definir la forma en que las empresas operan, se controlan y cómo interactúan con sus accionistas y demás partes interesadas dentro de la organización. Además, este gobierno sostiene como uno de sus principales objetivos, el transparentar la relación entre los accionistas y el consejo de administración. Para tales efectos, es necesaria la observación de una serie de principios básicos, cuyo objetivo es proteger los intereses de los accionistas y maximizar el valor de las empresas en el largo plazo

Dentro del tema de gobierno corporativo, el sistema de control interno es un eslabón básico para lograr que su infraestructura resulte altamente eficiente y efectiva.

Por consiguiente el Control interno lo define Moreno, E (2009) como:

"El sistema integrado por el esquema de organización y el conjunto de los planes, métodos, principios, normas, procedimientos y mecanismos de verificación y evaluación adoptados por una entidad, con el fin de procurar que todas las actividades, operaciones y actuaciones, así como la administración de la información y los recursos, se realicen de acuerdo con las normas constitucionales y legales vigentes, dentro de las políticas trazadas por la dirección y en atención a las metas u objetivos previstos."

Así mismo establece que los objetivos del control interno no son más que un plan de organización de todos los métodos coordinados con algunas de las siguientes finalidades:

- Proteger los recursos de la organización, buscando su adecuada administración ante posibles riesgos que los afecten;

- Garantizar la eficacia, la eficiencia y economía en todas las operaciones promoviendo y facilitando la ejecución de las funciones y actividades definidas para el logro de la misión institucional;

- Velar porque todas las actividades y recursos de la organización estén dirigidos al cumplimiento de los objetivos de la entidad;

- Garantizar la correcta evaluación y seguimiento de la gestión organizacional;

- Asegurar la oportunidad y confiabilidad de la información financiera y de sus registros;

- Definir y aplicar medidas para prevenir los riesgos, detectar y corregir las desviaciones que se presenten en la organización y que puedan afectar el logro de sus objetivos.

Todas las actividades de control que realice una empresa deben de ser apropiadas con el objetivo de minimizar riesgos.

Nuevamente López, V. (2009) establece que:

"Hablar del sistema de control interno implica hablar de una serie de procesos y ele- 
mentos que al ser implantados integralmente, permiten una seguridad razonable de que se logren los objetivos de la organización, los cuales pueden tener una naturaleza estratégica, administrativa u operativa."

Es decir, es una serie de referencias y mecanismos de mejores prácticas que se aplican a la planeación, organización, ejecución, dirección, información y seguimiento para darle certidumbre a la toma de decisiones, orientando a las organizaciones, con una seguridad razonable, al logro de sus objetivos, bajo un ambiente eficiente, ético, de cumplimiento, calidad y mejora continua.

Por lo tanto después de haber conceptualizado algunos indicadores como son gobierno corporativo, ética profesional, control interno, contabilidad desde un enfoque general e información financiera, que pretenden explicar el porqué de la contabilidad creativa, nos resta conceptualizar lo que corresponde a este término, de para lo cual de acuerdo a Bermudez, C. (2009), la define de la siguiente forma:

"A la información suministrada por los estados financieros sobre la situación de la empresa, hace necesario encontrar muchas veces una manipulación intencionada o natural de los resultados alcanzados, todo ello para mostrar una actitud positiva y atractiva a todos los usuarios externos ya sean inversionistas, acreedores, y prestamistas entre otros."

Este tipo de manipulación contable es lo que lleva el nombre de contabilidad creativa o también conocida como alisamiento del resultado o alisamiento del beneficio, y nace donde termina la uniformidad.

Para Pirela, W. (2009), la contabilidad creativa: Consiste fundamentalmente en aprovechar los vacíos de la normativa legal en cuanto a las diferentes posibilidades de elección con el propósito de transformar la información contable que se presentan en los estados financieros de las empresas.

Cabe hacer mención de algunas razones por las cuales las empresas o empresarios han optado por la utilización de dicha contabilidad son:

- El impacto que puede generar sobre los inversionistas en las distintas bolsas de valores donde participe la entidad.

- Expresar una tendencia financiera estable en relación a crecimiento, beneficios obtenidos.

- Reduciendo los niveles de endeudamiento, generando que aparentemente la expresa se encuentra expuesta a menores riesgos financieros.

- La aplicación de lagunas o ambigüedades de las leyes fiscales que benefician al empresario, con la disminución de pagos tributarios o algunos tipos de subsidios.

Por desgracia en la actualidad, prácticamente todas las empresas "maquillan las cuentas" y rara es la empresa que no la ha empleado, y muchas de ellas con el simple objetivo de subsistir.

\section{CONCLUSIÓN}

Una vez que se revisaron diversos conceptos y después de realizar un breve análisis, de diversos artículos sobre contabilidad creativa, contabilidad general, información financiera, ética profesional desde el enfoque contable y gobierno corporativo, se concluye diciendo que:

La contabilidad general ha sido considerada como disciplina muy antigua a través de la historia, esta ha evolucionado a medidas que se incrementó la complejidad de los sistemas económicos y sociales y los requerimientos a satisfacer. Dentro de las funciones 
básicas de la contabilidad encontramos que una que es vita y consiste en: Suministrar información financiera relevante y significativa, sobre las variaciones del patrimonio de un ente económico que permita una adecuada toma de decisiones.

La contabilidad, juega un papel efectivo $y$ decisivo en la sociedad y no debe perseguir objetivos independientes. Este proceso debe ser realizado de acuerdo con normas y procedimientos de carácter general o especifico, que son aplicados al registro de las operaciones y a la presentación de las cifras en los estados financieros.

Pero también es conocido que la demanda por nuevas áreas contables es clara y sólida, y la profesión debe necesariamente, dirigir sus esfuerzos a cumplir con los anhelos y aspiraciones de la sociedad. La contabilidad se encuentra en un proceso de adecuación, derivado de la globalización mundial.

Pero en últimas fechas, la expresión "contabilidad creativa" ha comenzado a formar parte del lenguaje contable, causando un gran impacto en el ámbito económico, financiero y gerencial. El fenómeno de la contabilidad creativa ha sido el resultado de la flexibilidad de ciertas normas contables la cual facilita la manipulación, engaño y tergiversación de la información financiera. Algunos autores lo atribuyen como una consecuencia de la crisis financiera que ha afectado a grandes compañías de ámbito internacional, dado por la intención de impresionar con estados financieros que proyecten una solidez y un crecimiento sostenido y en ascenso permanente de su patrimonio.

La contabilidad creativa ha cobrado especial relevancia, poniendo en duda los datos que se presentan, así como la credibilidad de diversos actores que intervienen en la generación y revisión de dicha información.
No es falta de organización, ni de control interno contable, interviene la ética profesional de quien se presta para la realización de esta práctica contable, que es en mucho casos originado inicialmente por la presión existente del inversionista frente al gerente de la compañía y este ultimo recurre a distorsionar los conceptos, principios, normas y todo método de la información contable para incrementar el nivel de resultados de la entidad.

Es importante mencionar que el uso de este tipo de contabilidad, tiene repercusiones importantes en los diversos interesados, por la toma de decisiones que realizan, derivada de la información generada por las empresas y adicionalmente cuando este tipo de empresas son descubiertas de las prácticas no tan legales, o aún siendo legales, pero demuestran que su información financiera no es relevante ni significativa, llegan a carecer de toda credibilidad y confianza, deteriorando su imagen crediticia, financiera. Un ejemplo de una situación real lo menciona Cardona, M. (2009)

"Casos como lo sucedido con el gigante de la eléctrica Enron, Worldcom, Tyco etc sacan a la luz pública los casos de corrupción practicados por estas multinacionales, sin medir el daño que causarían tanto a ahorradores que veían en sus estados financieros una empresa sólida con magnificas rentabilidades una buena opción de invertir sin mencionar la cantidad de trabajadores que serian despedidos y el daño tan grande hecho a la credibilidad de la información financiera, poniendo en crisis la realización de negocios ya que se generan en un ambiente caracterizado por las dudas, por una incertidumbre creciente surgida de las manipulaciones de la información con base en la cual los inversionistas tomaran sus decisiones." 
Se concluye diciendo a todos los contadores:

¿Vale la pena este tipo de prácticas contables que ponen en riesgo nuestra calidad moral y profesional por una ganancia adicional no tan bien generada, donde interponemos nuestra ética profesional y nuestra tranquilidad personal?

\section{REFERENCIAS BILIOGRÁFICAS}

1. Bermúdez, C. (2009). "Contabilidad creativa". Editorial El cid Editor p. 18; Argentina [En línea] disponible en http://site.ebrary.com/lib/uaehsp/ Doc?id=10316554\&ppg=5

[Accesado el día 02 de septiembre de 2011]

2. Cardona, M. (2009), "Confianza en la información contable.” Editorial El cid Editor p. 9; Argentina [En línea] disponible en http://site.ebrary.com/lib/uaehsp/ Doc?id=10316842\&ppg $=8 \quad$ [Accesado el día 01 de octubre de 2011]

3. Código de ética profesional del IMCP 2009, 8va. Edición.

4. Cruz, E. (2009) "El Futuro del control interno" Revista Contaduría Pública del mes de Febrero 2009, p.p. 44- 47 [En línea] disponible en http://ccpm.org.mx/ servicios/archivos/contaduria_publicaFEB09.pdf [ Accesado el día 29 de septiembre de 2011]

5. Galindo, M. (2008) "Diccionario de contabilidad, auditoría y control de gestión”, Editorial Ecobook, Editorial del Economista., España p. 249 ISBN 9788496877238

6. González, I. (2009) "Controles internos en la administración de riesgos." Revista
Contaduría Pública del mes de Febrero 2009, p.p. 10- 13 [En línea] disponible en http://ccpm.org.mx/servicios/archivos/contaduria_publicaFEB09.pdf [Accesado el día 20 de septiembre de 2011]

7. López, V. (2009) "Gobierno Corporativo y sistema de control interno", Revista Contaduría Pública del mes de Febrero 2009, p.p. 40- 43 [En línea] disponible en http://ccpm.org.mx/servicios/archivos/contaduria_publicaFEB09.pdf [Accesado el día 18 de septiembre de 2011]

8. Moreno, E. (2009) "Auditoria", Editorial El cid. Editor Pág. 58; Argentina [En línea] disponible en http://site.ebrary.com/lib/uaehsp/ Doc?id=10316831\&ppg=42 [Accesado el día 05 de octubre de 2011]

9. Pirela, W. (2009). "La contabilidad creativa: un maquillaje a los estados financieros". Editorial El cid E d i t o r . Argentina. p. 19 [En línea] disponible en http://site.ebrary.com/lib/uaehsp/ Doc?id=10316779\&ppg=5 [Accesado el día 12 de septiembre de 2011]

10. Ramos, R. y Becerra, P. (2009) "Historia de la contabilidad", Editorial de la Universidad de Santiago de Chile; Chile; p. 237 ISBN 9789563030792 [En línea] disponible en http://site.ebrary.com/ lib/uaehsp/Doc?id=10472800 [Accesado el día 05 de septiembre de 2011]

11. http://www.ccpm.org.mx

12. http://es.scribd.com/doc/47920621/ NIF-A-1-ESTRUCTURA-DE-LASNORMAS-DE-INFORMACION-FINANCIERA 